FOLIA

Amazónico

Revista del Instituto de Investigaciones

de la Amazonía Peruana

\title{
COMPOSICIÓN NUTRICIONAL Y CAPACIDAD ANTIOXIDANTE DE TRES ESPECIES DE SACHA INCHI Plukenetia spp. DE LA AMAZONÍA PERUANA
}

\author{
Jak BARDALES ARÉVALO ${ }^{1}$, Claudia MERINO ZEGARRA ${ }^{1}$, \\ Billy CABANILLAS AMADO ${ }^{1}$, Ángel Martín RODRÍGUEZ DEL-CASTILLO², \\ Gabriel VARGAS-ARANA ${ }^{1}$ \\ 1 Laboratorio de Química de Productos Naturales, Instituto de Investigaciones de la Amazonía \\ Peruana, Av. Abelardo Quiñones km 2.5, lquitos, Perú. \\ Correo electrónico: gvargas@iiap.gob.pe \\ 2 Laboratorio de Botánica Aplicada Jean-Christophe Pintaud, Instituto de Investigaciones de la \\ Amazonía Peruana, Jirón Belén Torres de Tello, Tarapoto, Perú.
}

\section{RESUMEN}

Se evaluó y se comparó la composición química y actividad antioxidante de tres especies de semillas de sachainchi (Plukenetia voluvilis, Plukenetia carolis-vegaey Plukenetia huayllabambana). Se realizaron los análisis de composición proximal, análisis de micro y macro nutrientes, propiedades fisicoquímicas y composición de ácidos grasos de los aceites, y la evaluación de la actividad antioxidante mediante los métodos de DPPH y ABTS. Los resultados mostraron una similitud entre $P$. carolis-vegae y $P$. huayllabambana, estas especies presentaron un alto contenido de aceites $(49,28$ y 48,82 \%, respectivamente) en comparación con $P$. voluvilis $(34,23 \%)$. Las tres especies presentaron similar perfil de ácidos grasos siendo el ácido linolénico (omega-3) el mayoritario (48,32-58,71\%). En el contenido de minerales el potasio fue el mineral más predominante en P. carolisvegae y $P$. huayllabambana $(686,04$ y $710,69 \mathrm{mg} / 100 \mathrm{~g}$, respectivamente), a diferencia de $P$. voluvilis que fue el calcio $(237,92 \mathrm{~m} / 100 \mathrm{~g})$. P. carolis-vegae fue la especie que presentó mayor actividad antioxidante (DPPH 2,30 $\mu \mathrm{mol}$ TE/g, ABTS 3,38 $\mu \mathrm{mol} \mathrm{TE} / \mathrm{g}$ ) correlacionándose con su contenido de fenoles totales (3,85 mg AG/100g). Estos resultados nos indican que P. carolis-vegae también pudiera ser aprovechado por la industria alimenticia y farmacéutica como lo es P. voluvilis, debido a que presenta buenas propiedades nutricionales.

PALABRAS CLAVES: Plukenetia carolis-vegae; Plukenetia huayllabambana; sacha inchi; actividad antioxidante; Amazonia peruana. 


\title{
NUTRITIONAL COMPOSITION AND ANTIOXIDANT CAPACITY OF THREE SPECIES OF SACHA INCHI Plukenetia spp. FROM PERUVIAN AMAZON
}

\begin{abstract}
Chemical composition and antioxidant activity of three species of sacha inchi seeds (Plukenetia voluvilis, Plukenetia carolis-vegae and Plukenetia huayllabambana) were evaluated and compared. The analyzes of proximal composition, analysis of micro and macro nutrients, physicochemical properties and fatty acid composition of the oils, and the evaluation of the antioxidant activity using the methods of DPPH and ABTS were performed. The results showed a similarity between $P$. carolis-vegae and $P$. huayllabambana, these species had a high oil content ( 49.28 and $48.82 \%$, respectively) compared to P. voluvilis (34.23\%). The three species presented a similar fatty acid profile, with linolenic acid (omega-3) being the majority (48.32-58.71\%). In the mineral content, potassium was the most predominant in P. carolis-vegae and P. huayllabambana (686.04 and $710.69 \mathrm{mg} / 100 \mathrm{~g}$, respectively), unlike P. voluvilis which was calcium (237, $92 \mathrm{~m}$ / 100g). P. carolis-vegae was the species that presented the highest antioxidant activity (DPPH $2.30 \mu \mathrm{mol} \mathrm{TE} / \mathrm{g}$, ABTS $3.38 \mu \mathrm{mol}$ TE/g) and correlated with its total phenolic content (3.85 mg AG / 100g). These results indicate that $P$. carolisvegae could also be used by the food and pharmaceutical industry as $P$. voluvilis, because it presents better nutritional properties.

KEYWORDS: Plukenetia carolis-vegae; Plukenetia huallabambana; sacha inchi; antioxidant activity; Peruvian Amazon
\end{abstract}




\section{INTRODUCCIÓN}

Sacha inchi (Plukenetia spp.), también conocido como maní del Inca, maní silvestre, maní sacha o maní de montaña, es una planta oleaginosa que pertenece a la familia Euphorbiaceae que crece en las tierras bajas de la Amazonía peruana. Ha sido cultivado y consumido durante siglos, tal y como lo demuestran las vasijas de barro con la forma de su fruto, que fueron encontradas en culturas pre-incaicas. Tradicionalmente forma parte de una variedad de platos indígenas de la Amazonia peruana (Nusselder \& Cloesen, 2015). El aceite (mezclado con harina) es usado con fines cosméticos por mujeres de las tribus Mayoruna, Campas, Huitotas, Shipibas, Yaguas y Bora. También se ha usado médicamente en el tratamiento de problemas reumáticos y dolores musculares (Hanssen \& Schmitz-Hübsch, 2011).

Por otro lado, el consumo moderado de antioxidantes tiene un efecto beneficioso en la salud, debido a que estas sustancias inactivan o neutralizan los radicales libres que se encuentran en desequilibrio y generan daño oxidativo (alteraciones estructurales y funcionales en las células), que se relaciona con el desarrollo de diferentes enfermedades crónicas como la diabetes mellitus, el cáncer y la enfermedad cardiovascular (Cuerda et al., 2011; López Ramírez \& Echeverri, 2007; Fu et al., 2011).

La semilla de sacha inchi, en especial la de $P$. volubilis, ofrece beneficios nutricionales interesantes, debido a su alto contenido de proteínas, tocoferoles, ácidos grasos poliinsaturados, además de un alto potencial como alternativa $y$ fuente novedosa de compuestos fenólicos y antioxidantes (Chirinos et al., 2013; Chirinos et al., 2016; Castaño et al., 2012; Gutiérrez et al., 2011).

LasespeciesP.carolis-vegaeyP.huayllabambana, anteriormente consideradas como variedades locales de $P$. volubilis, recientemente han sido reclasificadas y descritas como dos nuevas especies. P. carolis-vegae muestra características morfológicas similares a $P$. huayllabambana, pero difiere en el número de sépalos en las flores estaminadas, en el tamaño de hojas, filamentos y fruto Rodríguez et al., 2010; Bussmnn et al., 2013; Bussmann et al., 2009).

En estudios previos realizados a la semilla de P. huayllabambana, esta se destaca por su alto contenido de aceite, superior en casi un 9\% a P. volubilis, con una mayor proporción de ácido linolénico (omega-3); asimismo presenta un alto contenido proteico, y se han encontrado contenidos importantes de tocoferoles, fitoesteroles y compuestos fenólicos, siendo una fuente prometedora de aceite vegetal de alta calidad y de compuestos bioactivos, e interesante no solo desde el punto de vista nutricional, medicinal y cultural, sino también con enormes posibilidades para la industralización (Ruiz et al., 2013; Muñoz Jáuregui et al., 2013; Chasquibol et al., 2014., Chirinos et al., 2015). Con respecto a $P$. carolis-vegae, ésta al ser una especie recientemente descrita, hasta la fecha no existen estudios previos sobre su valor nutricional ni su potencial como antioxidante. El objetivo del presente trabajo fue determinar, y comparar entre ellas, el aporte nutricional y la capacidad antioxidante de las semillas de $P$. voluvilis, $P$. huayllabambana y $P$. carolis-vegae, como una nueva alternativa de alimento funcional $o$ nutracéutico.

\section{MATERIAL Y MÉTODOS}

\section{MATERIAL BIOLÓGICO}

Las muestras botánicas y semillas de Plukenetia carolis-vegae y Plukenetia huayllabambana fueron colectadas en las localidades de Monte 
Alegre y San Nicolás, respectivamente, ambas ubicadas en la provincia de Rodríguez de Mendoza, Región de Amazonas, Perú. P. voluvilis fue colectado en la localidad de Lamas, provincia de Lamas, región San Martín. Las muestras fueron puestas en congelación $\left(4^{\circ} \mathrm{C}\right)$ hasta su utilización. Muestras de cada especie fueron depositadas en el Herbario Amazonense de la Universidad Nacional de la Amazonía Peruana.

\section{ANÁLISIS PROXIMAL}

El análisis proximal de cada una de las muestras fue realizado según los métodos recomendados por la AOAC (2005). El contenido de humedad se determinó por secado de las muestras a $105^{\circ} \mathrm{C}$ durante $3 \mathrm{~h}$ en una estufa. Las cenizas fueron determinadas por incineración a $550^{\circ} \mathrm{C}$ durante $5 \mathrm{~h}$ en una mufla. El contenido total de grasas fue determinado por el método de soxhlet. El contenido de nitrógeno fue determinado usando el método kjeldahl y mulplicado por un factor $(6,25)$ para determinar el contenido de proteína cruda. Fibra por tratamiento con ácido y base. Carbohidratos por diferencia (100 - suma de los porcentajes de cada uno de los anteriores).

\section{ANÁLISIS DE MINERALES}

Se realizaron digestiones sucesivas con ácido clorhídrico $3 \mathrm{~N}$ a las cenizas obtenidas de las semillas molidas. Los minerales $(\mathrm{Na}, \mathrm{K}, \mathrm{Ca}, \mathrm{Mg}$, $\mathrm{Mn}, \mathrm{Fe}, \mathrm{Zn}$ y $\mathrm{Cu}$ ) se midieron por absorción atómica utilizando un espectrofotómetro Varian AA240, previamente calibrado con soluciones estándares que contienen cantidades conocidas de los minerales que se determinan, utilizando reactivos de grado analítico. Se utilizó dos tipos de llama, aire-acetileno y óxido nitrosoacetileno, este último solo para el análisis de calcio. Se emplearon lámparas de cátodo hueco monometálicas para cada elemento analizado (AOAC, 2005).

\section{ANÁLISIS FISICOQUÍMICOS Y COMPOSICIÓN} DE ÁCIDOS GRASOS DEL ACEITE

Los análisis fisicoquímicos del aceite extraído de las semillas, con éter de petróleo, fueron realizados de acuerdo a la metodología recomendada de la AOAC (2005). La composición de los ácidos grasos fue determinada por cromatografía gaseosa de acuerdo a la metodología empleada por Chririnos et al. (2015). Los ácidos grasos de las muestras de los aceites fueron derivatizados a ésteres volátiles y analizados en un equipo Varian 450-GC equipado con un detector de ionización de llama y autoinyector Varian CP8400. La columna usada fue una VF-WAXms, $60 \mathrm{~m}$ x 0,25 mm ID, 0,25 $\mu \mathrm{m}$, CP9207. La temperatura del horno se programó de la siguiente manera: desde $190^{\circ} \mathrm{C}$ (isotérmico durante 3 minutos) hasta $230^{\circ} \mathrm{C}$ a $1,3^{\circ} \mathrm{C} /$ minuto. Las temperaturas del inyector y del detector se ajustaron a $250^{\circ} \mathrm{C}$ y $270^{\circ} \mathrm{C}$ respectivamente. Se usó helio como gas portador. Los ácidos grasos esterificados fueron identificados y cuantificados por comparación con el tiempo de retención conocido de los estándares previamente inyectados.

\section{ACTIVIDAD ANTIOXIDANTE \\ Extracción de fenólicos}

$0,5 \mathrm{~g}$ de semilla fresca triturada de sacha inchi, se colocaron en un tubo para centrifuga a la cual se agregaron $5 \mathrm{~mL}$ de metanol acuoso al 80\%, la mezcla fue agitada en un vórtex durante 15 min. Seguidamente fue centrifugada a 5,000 rpm, durante 15 min a $5{ }^{\circ} \mathrm{C}$. El sobrenadante (extracto fenólico) fue almacenado a $-4{ }^{\circ} \mathrm{C}$ hasta su respectivo análisis.

\section{Método DPPH}

Se utilizó el método desarrollado por BrandWillians et al. (1995), con algunas modificaciones. A 3,9 mL de una solución del radical DPPH $\bullet(100$ $\mu \mathrm{M}$ ) disuelto en metanol al $80 \%$, se añade 0,1 
$\mathrm{mL}$ del extracto fenólico, filtrado previamente en un filtro de membrana $(0,45 \mu \mathrm{m})$, la mezcla se agitó vigorosamente y se dejó reposar en la oscuridad por 30 minutos a $25^{\circ} \mathrm{C}$. Transcurrido ese tiempo se leyó la absorbancia a 517 nm en un espectrofotómetro UV-visible Cary60. La concentración de DPPH• en el medio de reacción se calcula a partir de una curva de calibrado obtenida por regresión lineal. El control consistió en $0,1 \mathrm{~mL}$ de metanol acuoso al $80 \%$ y 3,9 mL de solución de DPPH• $(100 \mu \mathrm{M})$. Los resultados se expresan en TEAC, o sea, actividad antioxidante equivalente a Trolox ( $\mu$ mol Trolox/g de muestra peso fresco). El antioxidante sintético de referencia Trolox, a una concentración de 5-30 $\mu \mathrm{M}$ en disolución de metanol al $80 \%$, se ensaya en las mismas condiciones.

\section{Método ABTS}

Se utilizó en método desarrollado por Re et al. (1999). La reacción se inició con la adición de $1500 \mu \mathrm{l}$ de una solución ABTS $^{+\bullet}$ en buffer PBS $(0,70 \pm 0,02$ a $\lambda=734 \mathrm{~nm})$ a $500 \mu \mathrm{l}$ del extracto fenólico en una cubeta mantenida a 30 C. Se homogenizó y se dejó reaccionar por 7 minutos, seguidamente se hizo la lectura de absorbancia a una longitud de onda de $734 \mathrm{~nm}$, utilizando un espectrofotómetro UV-visible Cary60. Los resultados se expresan en TEAC ( $\mu$ mol Trolox/g de muestra peso fresco). La curva de calibración para el TEAC se construyó utilizando diferentes concentraciones de Trolox (4-14 $\mu \mathrm{M})$ en solución buffer PBS en las mismas condiciones.

\section{Determinación de fenoles totales}

Los fenoles totales fueron estimados mediante un método colorimétrico basado en los procedimientos descritos por Velioglu et al. (1998) con algunas modificaciones. Básicamente, $100 \mu \mathrm{l}$ del extracto fenólico fueron mezclados con $750 \mu \mathrm{l}$ del reactivo Foli-Ciocalteu diluido en proporción $1 / 10$ de agua Milli-Q. Después de 5 minutos en la oscuridad, $750 \mu \mathrm{l}$ de bicarbonato de sodio $(60 \mathrm{~g} / \mathrm{l})$ fueron agregados a la mezcla. Los tubos fueron mantenidos en la oscuridad por 90 minutos a 30 ${ }^{\circ} \mathrm{C}$, seguidamente se leyó la absorbancia a 725 nm. El ácido gálico (10-100 $\mu \mathrm{g}$ ) fue utilizado para la construcción de la curva estándar. Los resultados se expresan como mg de ácido gálico/ g de muestra de peso fresco.

\section{RESULTADOS Y DISCUSIÓN}

\section{ANÁLISIS PROXIMAL}

Los resultados del análisis proximal (tabla 1) muestran que las semillas de $P$. carolis-vegae y $P$. huayllabambana destacan por su alto contenido de grasas $(49,28$ y 48,82 \%, respectivamente) en comparación con P. voluvilis (34,23\%), resultados similares, para esta diferencia, lo reportó Ruiz et al. (2013), cuando compara el porcentaje de grasas, en base seca, de las semillas de $P$. huayllabambana $(54,3 \%)$ con $P$. voluvilis $(49,0 \%)$. Así mismo, nuestros resultados coinciden con lo reportado por Muñoz Jáuregui et al. (2013), para el contenido de grasas de dos accesiones de $P$. huayllabambana (45,87 y 48,84\%). En general,

Tabla 1. Análisis proximal de las tres especies de sacha inchi

\begin{tabular}{|l|c|c|c|}
\hline \multicolumn{1}{|c|}{ Análisis } & P. voluvilis & P. carolis-vegae & P. huayllabambana \\
\hline \% Humedad & $6,28 \pm 0,03$ & $6,90 \pm 0,06$ & $7,68 \pm 0,09$ \\
\hline \% Ceniza & $2,53 \pm 0,01$ & $2,59 \pm 0,01$ & $2,80 \pm 0,06$ \\
\hline \% Grasa & $34,23 \pm 0,49$ & $49,28 \pm 0,49$ & $48,82 \pm 0,55$ \\
\hline \% Proteína & $16,43 \pm 0,01$ & $14,26 \pm 0,14$ & $8,83 \pm 0,13$ \\
\hline \% Fibra cruda & $8,64 \pm 0,25$ & $3,33 \pm 0,11$ & $3,58 \pm 0,14$ \\
\hline \% Carbohidratos & 31,89 & 23,64 & 28,29 \\
\hline
\end{tabular}


según Wang et al. (2018), las tres especies se encuentran en el rango estimado de porcentaje de grasas para semillas de sacha inchi $(33,4$ y $55,3 \%)$. Además del contenido de aceite, las semillas contienen un moderado porcentaje de proteína, siendo en este caso la $P$. voluvilis $(16,43 \%)$ la de mayor contenido proteico, coincidiendo con lo reportado por Ruiz et al. (2013). El contenido proteico encontrado para estas tres especies de sacha inchi es menor que el rango reportado por Wang et al. (2018) para P. voluvilis (24.2-27.0\%).

\section{ANÁLISIS DE MINERALES}

En la tabla 2, se muestra el contenido de minerales presentes en las tres especies de sacha inchi. Para el caso de $P$. carolis-vegae y P. huayllabambana,

obtenido por Gutiérrez et al. (2011), a diferencia de lo reportado por Muñoz Jauregui et al. (2013) para $P$. huayllabambana, donde los valores son superiores para Hierro $(44,06 \mathrm{mg} / 100 \mathrm{~g})$, Zinc $(38,78 \mathrm{mg} / 100 \mathrm{~g})$ y Magnesio $(2446,09 \mathrm{mg} / 100 \mathrm{~g})$, esto se puede deber a que tal vez lo expresa en base a la muestra seca y desengrasada.

\section{ANÁLISIS FISICOQUÍMICOS Y COMPOSICIÓN} DE ÁCIDOS GRASOS DE LOS ACEITES

En la tabla 3, se observa que las tres especies presentaron valores similares para las propiedades fisicoquímicas de los aceites. Los valores de índice de peróxidos se encuentran por debajo del límite permisible $\left(10\right.$ meq $\mathrm{O}_{2} / \mathrm{kg}$ ) recomendado por el Codex Alimentario, siendo el valor de $P$. carolis-vegae $\left(4,04\right.$ meqO $_{2} /$

Tabla 2. Contenido de minerales en tres especies de sacha inchi

\begin{tabular}{|l|c|c|c|}
\hline $\begin{array}{c}\text { Minerales } \\
\text { (mg/100g MF) }\end{array}$ & P. voluvilis & P. carolis-vegae & P. huayllabambana \\
\hline Sodio & $41,38 \pm 0.13$ & $120,29 \pm 2,63$ & $118,14 \pm 15,61$ \\
\hline Potasio & $33,30 \pm 1.90$ & $686,04 \pm 18,05$ & $710,69 \pm 17,73$ \\
\hline Calcio & $237,92 \pm 2.93$ & $120,63 \pm 1,95$ & $77,00 \pm 8,51$ \\
\hline Magnesio & $97,49 \pm 4.41$ & $282,58 \pm 1,75$ & $244,56 \pm 8,33$ \\
\hline Manganeso & $1,26 \pm 0.03$ & $0,53 \pm 0,03$ & $0,57 \pm 0,02$ \\
\hline Hierro & $5,01 \pm 0.18$ & $3,83 \pm 0,05$ & $3,61 \pm 0,03$ \\
\hline Zinc & $5.42 \pm 0.02$ & $5,11 \pm 0,46$ & $5,46 \pm 0,53$ \\
\hline Cobre & $0.97 \pm 0.02$ & $3,12 \pm 0,15$ & $3,78 \pm 0,04$ \\
\hline
\end{tabular}
$\mathrm{kg}$ ) menor que para las otras dos especies de sacha inchi. Los valores ácidos grasos libres de las tres especies están por encima de lo recomendado para un producto alimenticio, según Carvalho et al. (2006) no deben pasar de 0,60\%. Chirinos et al. (2015) reporta valores menores de ácidos grasos libres e índice de peróxidos tanto para $P$. voluvilis $(0,19 \%$ ácido oleico, 2,90 meq $_{2} / \mathrm{kg}$ ) como para

el potasio fue el mineral más predominante (686,04 y 710,69 mg/100g, respectivamente), a diferencia de $P$. voluvilis que presentó un valor bajo $(33,30 \mathrm{mg} / 100 \mathrm{~g})$, en comparación con lo reportado por Gutiérrez et al. (2011) para semillas de $P$. voluvilis procedentes de Colombia $(556,30 \mathrm{mg} / 100 \mathrm{~g})$. Las tres especies mostraron un alto contenido de Zinc, que varía entre 5,11 y 5,46 mg/100g, y bajos contenidos de Cobre, Hierro y Sodio; estos valores se corroboran lo
P. huayllabambana (0,15 \% ácido oleico, 1,83 meq $\mathrm{O}_{2} / \mathrm{kg}$ ). El índice de yodo, índice de saponificación y densidad de las tres especies fueron similares a los reportados por Gutiérrez et al. (2011) (193,1 $\mathrm{g} \mathrm{I}_{2} / 100 \mathrm{~g}, 185,2 \mathrm{mg} \mathrm{KOH} / \mathrm{g}, 0,9187 \mathrm{~g} / \mathrm{cm}^{3}$, respectivamente).

\section{COMPOSICIÓN DE ÁCIDOS GRASOS}

La composición de los ácidos grasos se muestra en la tabla 4, las tres especies presentan un 
Tabla 3. Análisis fisicoquímicos de las tres especies de sacha inchi

\begin{tabular}{|l|c|c|c|}
\hline \multicolumn{1}{|c|}{$\begin{array}{c}\text { Análisis fisicoquímicos } \\
\text { del aceite }\end{array}$} & P. voluvilis & P. carolis-vegae & $P$. huayllabambana \\
\hline $\begin{array}{l}\text { Ácidos grasos libres } \\
\text { (\% ácido oleico) }\end{array}$ & $0.91 \pm 0.01$ & $0.80 \pm 0.01$ & $0.86 \pm 0.01$ \\
\hline Índice de saponificación $(\mathrm{mg} \mathrm{KOH/g)}$ & $185.45 \pm 0.63$ & $191.13 \pm 0.32$ & $185.09 \pm 0.32$ \\
\hline Índice de Peróxido $\left(\mathrm{meq} \mathrm{O}_{2} / \mathrm{Kg}\right)$ & $8.09 \pm 0.01$ & $4.04 \pm 0.01$ & $6.06 \pm 0.01$ \\
\hline Índice de yodo $\left(\mathrm{g} \mathrm{I}_{2} / 100 \mathrm{~g}\right)$ & $193.90 \pm 0.05$ & $199.07 \pm 0.01$ & $196.90 \pm 0.06$ \\
\hline Determinación de fósforo $(\mathrm{ppm})$ & $0,27 \pm 0,02$ & $0,16 \pm 0,02$ & $0,17 \pm 0,01$ \\
\hline Materia insaponificable $(\%)$ & $0.17 \pm 0.01$ & $0.32 \pm 0.01$ & $0.20 \pm 0.01$ \\
\hline Densidad $\left(\mathrm{g} / \mathrm{cm}^{3}\right)$ & $0.9234 \pm 0.01$ & $0.9252 \pm 0.01$ & $0.9288 \pm 0.01$ \\
\hline
\end{tabular}

Tabla 4. Composición de ácidos grasos en tres especies de Sacha inchi

\begin{tabular}{|l|c|c|c|}
\hline \multicolumn{1}{|c|}{ Ácidos grasos } & P. voluvilis & P. carolis-vegae & P. huayllabambana \\
\hline Ácido palmítico (C16:0) & $3,98 \pm 0,05$ & $4,82 \pm 0,04$ & $4,45 \pm 0,03$ \\
\hline Ácido esteárico (C18:0) & $2,88 \pm 0,04$ & $1,78 \pm 0,04$ & $1,91 \pm 0,05$ \\
\hline Ácido oleico (C18:1) & $8,47 \pm 0,03$ & $8,23 \pm 0,02$ & $8,30 \pm 0,04$ \\
\hline Ácido vaccénico (C18:1) & $0,58 \pm 0,04$ & $0,80 \pm 0,02$ & $0,73 \pm 0,02$ \\
\hline Ácido linoleico (C:18:2) & $35,78 \pm 0,04$ & $27,30 \pm 0,10$ & $25,90 \pm 0,07$ \\
\hline Ácido linolénico (C18:3) & $48,32 \pm 0,09$ & $57,06 \pm 0,10$ & $58,71 \pm 0,14$ \\
\hline \% AG Saturados & 6,86 & 6,60 & 6,36 \\
\hline \% AG Insaturados & 93,14 & 93,40 & 93,64 \\
\hline
\end{tabular}

alto contenido de ácidos grasos insaturados $P$. voluvilis $(93,14 \%), \quad P$. carolis-vegae $(93,40 \%)$ y $P$. huayllabambana $(93,64 \%)$, siendo los ácidos linolénico y linoleico los mayoritarios. $P$. huayllabambana y $P$. carolis-vegae muestran un alto contenido deácidolinolénico (58,71\% y 57,06, respectivamente), siendo estos valores mayores a los reportados por Ruiz et al. (2013) y Muñoz Jáuregui et al. (2013) para P. huayllabambana (53,9\% y $52,67 \%$, respectivamente), y también mayores al rango reportado por Wang et al. 2018 para aceites de $P$. voluvilis (45,20-50,41\%). $P$. voluvilis presentó el mayor contenido de ácido linoleico de las tres especies $(35,78 \%)$, similar a lo reportado por Ruiz et al. (2013) para P. voluvilis $(34,6 \%)$.

\section{ACTIVIDAD ANTIOXIDANTE}

Los resultados de actividad antioxidante para las tres especies de sacha inchi presentan valores similares, siendo la especie P. carolis-vegae la que presentó mayor actividad antioxidante en las dos metodologías evaluadas DPPH $(2,30 \mu \mathrm{mol}$ TE/g) y ABTS (3,38 $\mu \mathrm{mol} \mathrm{TE} / \mathrm{g})$; estos valores se encuentran relacionados con el contenido de fenoles totales $(3,85 \mathrm{mg} \mathrm{AG/g})$. Cabe resaltar 
Tabla 5. Actividad antioxidante y contenido de fenoles totales de tres especies de Sacha inchi

\begin{tabular}{|l|c|c|c|}
\hline \multicolumn{1}{|c|}{ Actividad antioxidante } & P. voluvilis & P. carolis-vegae & P. huayllabambana \\
\hline DPPH ( $\mu \mathrm{mol}$ TE/g MF) & $2.00 \pm 0.03$ & $2.30 \pm 0.01$ & $2.12 \pm 0.01$ \\
\hline ABTS ( $\mu \mathrm{mol}$ TE/g MF) & $2.90 \pm 0.02$ & $3.38 \pm 0.04$ & $2.63 \pm 0.03$ \\
\hline Fenoles Totales $(\mathrm{mg} \mathrm{AG} \mathrm{/g} \mathrm{MF)}$ & $2.98 \pm 0.03$ & $3.85 \pm 0.07$ & $3.22 \pm 0.04$ \\
\hline
\end{tabular}

que resaltar que las actividades antioxidantes presentadas por estas tres especies son mayores a lo reportado para el copoazú (DPPH 0,73 $\mu \mathrm{mol}$ TE/g; ABTS 2,0 $\mu \mathrm{mol} \mathrm{TE} / \mathrm{g}$ ) (Kuskoski et al., 2005), y menores a lo reportado por Cisneros et al. (2014) para el aceite de P. voluvilis (DPPH, 18,2 $\mu \mathrm{mol} \mathrm{TE} / \mathrm{g}$ aceite). La actividad antioxidante de las semillas o del aceite de las semillas depende mucho del tipo de tratamiento térmico que se les aplica, cuanto más tostado se encuentren la semilla mayor será su actividad antioxidante (Cisneros et al., 2014; Stervoba et al., 2017).

\section{CONCLUSIONES}

De la comparación entre semillas de sacha inchi se puede determinar que Plukenetia carolisvegae y Plukenetia huayllabambana presentan una similar composición química y actividad antioxidante; ambas tienen un alto contenido de aceites, superior a Plukenetia voluvilis, con elevadas concentraciones de ácidos grasos insaturados, constituidos principalmente por los ácidos linolénico y linoleico, siendo el ácido linolénico (omega-3) el de mayor contenido en $P$. carolis-vegae y P. huayllabambana. Las semillas de P. carolis-vegae y P. huayllabambana presentan un gran aporte de minerales con potasio, zinc y magnesio. Debido a la creciente demanda de alimentos funcionales y productos nutracéuticos, $P$. carolis-vegae también es una fuente invaluable para la industria de alimentos y farmacéutica.

\section{BIBLIOGRAFÍA CITADA}

Association of Official Agricultural Chemists. 2005. Official Methods of Analysis of the AOAC. 18 th ed. Washington, D.C., The Association.

Brand-Willian, W.; Cuvelier. M.E.; Berset, C. 1995. Use of free radical method to evaluated antioxidant activity. Lebensmittel-Wissenschaft und Technologie, 22: 25-30.

Bussmann, R.S.; Téllez, C.; Glenn, A. 2009. Plukenetia huayllabambana sp. nov (Euphorbiaceae) from the upper Amazon of Peru. Nordic Journal of Botany, 27: 313-315.

Bussmann, R.W.; Zambrana, N.P.; Téllez, C. 2013. Plukenetia carolis-vegae (Euphorbiaceae) A New Useful Species from Northern Peru. Economic Botany, 67: 387-392.

Castaño, D.L.; Valencia, M.P.; Murillo, E.; Mendez, J.J.; Joli, J.E. 2012. Fatty acid composition of inca peanut (Plukenetia volubilis Linneo) and its relationship with vegetal bioactivity. Revista Chilena de Nutrición, 39: 45-52.

Carvalho, I. S.; Miranda, I., Pereira, H. 2006. Evaluation of oil composition of some crops suitable from human nutrition. Industrial cops and Products, 24: 75-78.

Chasquibol, N.A.; Del Águila, C.; Yácono, J.C.; Guinda, A.; Moreda, W.; Gómez-Coca, R.B.; Pérez-Camino, C. 2014. Characterization of Glyceridic and Unsaponifiable Compounds of Sacha Inchi (Plukenetia huayllabambana L.) Oils. Journal of Agricultural and Food Chemistry, 62: 10162-10169. 
Chirinos, R.; Zuloeta, G.; Pedreschi, R.; Mignolet, E.; Larondelle, Y.; Campos, D. 2013. Sacha inchi (Plukenetia volubilis): A seed source of polyunsaturated fatty acids, tocopherols, phytosterols, phenolic compounds and antioxidant capacity. Food Chemistry, 141: 1732-1739.

Chirinos, R.; Pedreschi, R.; Domínguez, G.; Campos, D. 2015. Comparison of the physico-chemical and phytochemical characteristics of the oil of two Plukenetia species. Food Chemistry, 173: 1203-1206.

Chirinos, R.; Necochea, O.; Pedreschi, R.; Campos, D. 2016. Sacha inchi (Plukenetia volubilis L.) shell: an alternative source of phenolic compounds and antioxidants. International Journal of Food Science \& Technology, 51: 986993.

Cisneros, F. H.; Paredes, D.; Arana, A.; CisnerosZevallos, L. 2014. Chemical composition, oxidative stability and antioxidant capacity of oil extracted from roasted seed of Sacha-Inchi (Plukenetia voluvilis L.). Journal of Agricultual and Food Chemistry, 62: 5191-5197.

Cuerda, C.; Luengo, L.M.; Valero, M.A.; Vidal, A.; Burgo, R.; Calvo, F.L.; Martínez, C. 2011. Antioxidants and diabetes mellitus: review of the evidence. Nutrición Hospitalaria, 26: 6878.

Fu, L.; Xu, B.T.; Gan, R.Y.; Zhang, Y.; Xia, E.Q.; Li, H.B. 2011. Antioxidant capacities and total phenolic contents of 62 fruits. Food Chemistry, 129: 345-350.

Gutiérrez, L-F.; Rosada, L-M.; Jiménez, A. 2011. Chemical composition of Sacha Inchi (Plukenetia volubilis L.) seeds and characteristics of their lipid fraction. Grasas $y$ aceites, 62: 76-83.

Hanssen, H-P.; Schmitz-Hübsch, M. 2011. Sacha Inchi (Plukenetia volubilis L.) Nut Oil and Its Therapeutic and Nutritional Uses. In: Preedy, V.R.; Watson, R.R.; Patel, V.B. (Eds). Nuts and
Seeds in Health and Disease Prevention. Elsevier. p. 991-994.

Kuskoski, E. M.; Asuero, A. G.; Troncoso, A. M.; Mancini-Filho, J.; Fett, R. 2005. Aplicación de diversos métodos químicos para determinar la actividad antioxidante en pulpa de frutos. Ciencia e Tecnología de Alimentos, 25 (4): 726732.

López Ramírez, R.; Echeverri, F. 2007. ¿Son seguros y efectivos los antioxidantes? Scientia Et Technica, 13: 41-44.

Muñoz Jáuregui, A.M.; Alvarado-Urtiz, C.; Casteña, B.; Lizaraso, F.; Barnett, E.; Cárdenas, L.; Manco, E. 2013. Estudio nutricional de Plukenetia huayllabambana sp. Nov. Revista de la Sociedad Química del Peru, 79: 47-56.

Nusselder, H.; Cloesen, P. 2015. El cultivo de semillas nobles: ¿sacha inchi de la amazonía a la cuenca caribeña? In: van Dijck, P.; Nusselder, H. (Eds). Un recorrido por líneas locales: Aportes para políticas públicas en el sector rural de Centroamérica, el Caribe y la Región Andina. [Internet]. San José (CR). Centro de Estudios para el Desarrollo Rural. Available from: (http://cdr.or.cr/libro/completo.pdf). Acceso: 02/03/2019.

Re, R.; Pellegrini, N.; Proteggente, A.; Pannala, A.; Yang, M.; Rice-Evans, C. 1999. Antioxidant activity applying an improved ABTS radical cation decolorization assay. Free Radical Biology and Medicine, 26: 1231-1237.

Rodríguez, A.; Corazon-Guivin, M.; Cachique, D.; Mejía, K.; Del Castillo, D.; Renno, J-F.; GarcíaDávila, C. 2010. Differentiation morphological and by Inter simple sequence repeats (ISSR) of species of genus Plukenetia (Euphorbiaceae) from Peruvian Amazon: suggestion for a new species. Revista Peruana de Biología, 17: 325330.

Ruiz, C.; Diaz, C.; Anaya, J.; Rojas, R. 2013. Análisis proximal, antinutrientes, perfil de ácidos grasos y aminoácidos de semillas y tortas de 
2 especies de sacha inchi (Plukenetia volubilis y Plukenetia huayllabambana). Revista de la Sociedad Química del Perú, 79: 29-36.Sterbova, L.; Cepkova, P. H.; Viehmannova, I.; Cachique, D. 2017. Effect of thermal processin on phenolic content, tocopherols and antioxidant activity of Sacha inchi kernels. Journal of Food Processing and Preservation, 41: 1-8.
Velioglu, Y. S.; Mazza, G.; Gao, L.; Oomah, B.D. 1998. Antioxidant activity and total phenolics in selected fruits, vegetables, and grain products. Journal of Agricultural and Food Chemistry, 46: 4113-4117.

Wang, S.; Zhu, F.; Kakuda, Y. 2018. Sacha inchi (Plukenetia volubilis L.): Nutritional composition, biological activity, and uses. Food Chemistry, 265: 316-328.

Recibido: 5 de julio de 2019 Aceptado para publicación: 19 de agosto de 2019 